\title{
A phase II study of insulin-like growth factor receptor inhibition with nordihydroguaiaretic acid in men with non-metastatic hormone-sensitive prostate cancer
}

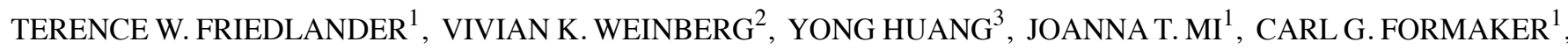 \\ ERIC J. SMALL ${ }^{1}$, ANDREA L. HARZSTARK ${ }^{1}$, AMY M. LIN ${ }^{1}$, LAWRENCE FONG ${ }^{1}$ and CHARLES J. RYAN ${ }^{1}$ \\ ${ }^{1}$ Genitourinary Medical Oncology Program, and ${ }^{2}$ Biostatistics and Computational Biology Core, Helen Diller \\ Family Comprehensive Cancer Center, University of California at San Francisco; ${ }^{3}$ Department of Bioengineering \\ and Therapeutic Sciences, University of California at San Francisco Medical Center, San Francisco, CA, USA
}

Received June 29, 2011; Accepted August 16, 2011

DOI: 10.3892/or.2011.1487

\begin{abstract}
Insulin-like growth factor (IGF)-mediated signaling is a newly recognized clinical target in prostate cancer, and it is hypothesized that blockade of the IGF receptor (IGF1R) will impair downstream signaling and slow tumor growth. In this study the efficacy of nordihydroguaiaretic acid (NDGA), a small molecule inhibitor of the IGF-1R, was prospectively evaluated in patients with non-metastatic hormone-sensitive prostate cancer (HSPC). Eligible patients had non-metastatic HSPC with a rising prostate-specific antigen (PSA) and a normal testosterone level. NDGA $2000 \mathrm{mg}$ was given orally daily in 28 day cycles and treatment continued until PSA progression or toxicity. Accrual was stopped early after a pre-planned interim analysis showed no significant PSA declines after 3 cycles of
\end{abstract}

Correspondence to: Dr Terence Friedlander, Box 1711, 1600 Divisadaro Street, University of California, San Francisco, San Francisco, CA 94115-1711, USA

E-mail: terence.friedlander@ucsf.edu

Abbreviations: ALT, alanine transaminase; AST, aspartate transaminase; $\mathrm{AR}$, androgen receptor; $\mathrm{C}_{\max }$, maximum concentration; $\mathrm{CT}$, computerized tomography; CTC, common toxicity criteria; $\mathrm{CV}$, coefficient of variation; GH, growth hormone; HER2, human epidermal growth factor receptor 2; HSPC, hormone-sensitive prostate cancer; IGF, insulin-like growth factor; IGF-1, insulin-like growth factor 1; IGFBP, insulin-like growth factor binding protein; IGF1R, insulin-like growth factor receptor; IRB, institutional review board; LFT, liver function tests; LOQ, limit of quantitation; MRI, magnetic resonance imaging; NDGA, nordihydroguaiaretic acid; $\mathrm{pK}$, pharmacokinetic; PSA, prostate-specific antigen; PSADT, prostatespecific antigen doubling time; RE, relative error; SD, standard deviation

Key words: prostate cancer, hormone-sensitive prostate cancer, rising prostate-specific antigen, insulin-like growth factor, insulin-like growth factor receptor, nordihydroguaiaretic acid, nordihydroguaiaretic acid treatment among the first 12 patients enrolled. Median time on treatment was 9 cycles (range 2-19) for 11 patients now off study; 1 patient continues to receive therapy and has been on study for 29 months. Seven patients experienced non-sustained declines in PSA ranging from 1.9 to $15.8 \%$ of baseline. PSADT lengthened by a median of 1.4 months for all evaluable patients when compared to pretreatment PSADT (range -6.1 to +19.8 months). Grade 3 events were rare and included nausea/ vomiting, syncope due to dehydration, and elevated liver function tests in 1 patient, and cognitive disturbance in another patient. NDGA therapy lengthens median PSADT but does not induce significant PSA declines. Further study may require a placebo-control to determine if changes in PSADT are drug related.

\section{Introduction}

The IGF signaling pathway is frequently activated in human malignancies and is currently under investigation in prostate cancer (1-4). Binding of circulating IGF-1 ligand to the membrane-bound receptor IGF1R induces activation of AKT and the Ras-PI3K-MAP kinase pathway, which in turn upregulates a diverse set of cellular and nuclear proteins, including transcription factors necessary for growth and survival (5). Engagement of IGFR1 can also influence hypoxia signaling, protease secretion, and can increase cell motility and adhesion, leading to an increased potential for invasion and metastasis (6).

In prostate cancer the IGF pathway interacts with the androgen receptor (AR) axis in multiple ways. IGF levels are increased in patients with prostate cancer, and progression from androgen sensitivity to androgen resistance is associated with an increase in IGF $(7,8)$. AR-mediated gene expression can be induced by IGF-1 even in the absence of androgen, and suppression of IGF1R expression inhibits tumor growth $(9,10)$. Additionally IGF1R is present in the tumor microenvironment, suggesting the possibility of a paracrine signaling loop that could be broken by an IGFR1 inhibitor (11). Taken together these data suggest that effective suppression of the IGF axis may lead to an inhibition of growth of prostate tumors. 
NDGA is a butanediol isolated from the chaparral bush Larrea tridentata of the southwestern United States and in Mexico. Extracts of the leaf and/or stem have been taken orally by the Pima Indians and other local cultures to treat diabetes mellitus and hepatitis, among other conditions (12). Functionally, NDGA is a small molecule inhibitor of multiple tyrosine kinases including the IGF1R and the human epidermal growth factor receptor 2 (HER2) (13). In preclinical studies NDGA was shown to suppress the anti-apoptotic signaling pathway downstream of IGF1R and to inhibit the in vitro growth of breast cancer cells (14). In prostate cancer cell lines NGDA rapidly inhibits the autophosphorylation of IGF1R induced by IGF-1 and leads to growth arrest of androgen-stimulated prostate cancer cells, independent of direct interaction with the androgen receptor $(15,16)$.

Based on this preclinical work, NDGA was evaluated in a phase I study of 15 men with non-metastatic hormone-sensitive $(n=11)$ and castration-resistant $(n=4)$ prostate cancer and was shown to be well tolerated. PSA declines and greater than 3-fold increases in PSADT were observed in $13 \%$ and $40 \%$ of subjects respectively, independent of changes in circulating androgen levels (17). Because it is well recognized that castrating therapies have deleterious effects on cardiac, metabolic and bone health $(18,19)$, NDGA was evaluated in this study as an alternative to androgen deprivation therapy for men with biochemically relapsed disease. The goals of this current study were to explore whether suppression of the IGF axis with NDGA was associated with PSA responses in men with non-metastatic HSPC, to determine the extent to which NDGA affected circulating androgen levels, and to assess the safety of this approach.

\section{Materials and methods}

Patient population. Eligible patients included men with biochemically recurrent non-metastatic prostate adenocarcinoma despite prior brachytherapy, external beam radiotherapy, or radical prostatectomy. Recurrence was defined as a serially rising PSA $\geq 1.0 \mathrm{ng} / \mathrm{ml}$ on at least 2 occasions for patients who underwent prostatectomy, and was defined as the nadir PSA value plus $2 \mathrm{ng} / \mathrm{ml}$ (Phoenix criteria) (20), for patients who received radiation therapy. Patients with local-only recurrence were excluded. Because androgen deprivation therapy is the standard of care for men with a rapidly rising PSA after definitive therapy, only subjects with a PSADT between 6 and 24 months based on four or more readings at least 2 weeks apart during a time period in which no other therapy was utilized were eligible. Patients who received neoadjuvant, concurrent and/or adjuvant hormonal therapy with radiotherapy, as well as patients who received adjuvant or salvage radiotherapy after prostatectomy, were included as long as at least 6 months had elapsed since hormonal therapy and the testosterone level was $\geq 250 \mathrm{ng} / \mathrm{dl}$ at enrollment. Karnofsky performance status $\geq 70 \%$ and normal renal, hematologic and liver function were required. Due to the potential for increases in serum glucose with NDGA therapy, subjects with diabetes mellitus or a history of treatment with insulin or an oral hypoglycemic agent were excluded. No concomitant 5- $\alpha$ reductase inhibitor, herbal, or other supplemental treatments intended to lower PSA were allowed. The study was approved by the UCSF Institutional Review Board (IRB) and all patients provided written informed consent.
Study therapy. Therapy consisted of NDGA $2000 \mathrm{mg}$ given orally daily in 3 divided doses $(750 \mathrm{mg}$ in the morning and in the evening, and $500 \mathrm{mg}$ at midday) over a 28-day cycle. Baseline imaging consisted of a radionuclide bone scan and computerized tomography (CT) or magnetic resonance imaging (MRI) of the abdomen and pelvis. Patients underwent repeat radiographic evaluation only at disease progression. PSA response was defined as a PSA decline of at least $50 \%$ from baseline confirmed by a second measurement at least 2 weeks later. Disease progression was defined by consensus criteria, which required a $25 \%$ increase in PSA over the nadir or baseline (whichever was lower) and a minimum increase in PSA level by at least $5 \mathrm{ng} / \mathrm{ml}$ (21). Patients were evaluated monthly for toxicity, which was graded according to common toxicity criteria (CTC), version $3.0(22)$.

Assay methods. PSA and testosterone were measured by chemiluminescent immunoassay on the Immulite 2000 analyzer (Siemens Healthcare Diagnostics, Deerfield,IL). Dihydrotestosterone and androstenedione were measured by radioimmunoassay (Quest Diagnostics, San Juan Capistrano, CA).

Pharmacokinetics. As a detailed pharmacokinetic (pK) study was not performed in the phase I study of NDGA and prior $\mathrm{pK}$ studies could not separate NDGA from its glucuronidated and sulfated metabolites, a pharmacokinetic analysis was included in this study. The first twelve patients enrolled were treated with a single 750-mg oral dose of NDGA on cycle 1 day 1 (fed for the first six subjects and fasting for the second six subjects) with measurements of plasma levels of NDGA and its metabolites obtained at zero, $30 \mathrm{~min}, 1,2,4$ and $8 \mathrm{~h}$ after the single dosage with a \pm 15 -min window for blood sampling. Steady state $\mathrm{pK}$ of NDGA and its metabolites were then measured on day 1 of each subsequent cycle for all patients. In accordance with published guidelines, fed patients were provided a breakfast containing 800-1000 calories with $\sim 50 \%$ of calories derived from fat (23). The 750-mg dose was chosen for $\mathrm{pK}$ analysis as this is the maximum dosage that subjects took at any one time point during the day.

For pK analysis blood samples were collected into heparinized tubes. Within 10 min of collection the plasma fraction was separated by centrifugation. After separation $0.1 \mathrm{M}$ of ascorbic acid was added immediately to the plasma fraction at a plasma:ascorbic acid ratio of 9:1. Samples were then immediatedly stored at $-70^{\circ} \mathrm{C}$ until analysis. Batched samples were analyzed by a liquid chromatography/mass spectrometric (LC/ $\mathrm{MS} / \mathrm{MS}$ ) procedure in a Micromass Quattro Ultima system (Waters Corp., Milford, MA) at the UCSF Drug Studies Unit according to a protocol developed by the UCSF Drug Studies Unit Analytical Division.

The assay used to determine NGDA concentration was validated using ascorbic acid-treated human plasma $(0.1 \mathrm{ml}$ of $0.1 \mathrm{M}$ ascorbic acid to $0.9 \mathrm{ml}$ plasma), which was then spiked with drug for the concentration range of 1.00-100 ng/ ml. NDGA in plasma was extracted with methyl t-butyl ether and then evaporated by $\mathrm{N} 2$ gas, reconstituted with $0.15 \mathrm{ml}$ of methanol and analyzed by LC/MS/MS in electrospray negative ionization mode. The multiple reaction monitor was set at $301.2-122.2 \mathrm{~m} / \mathrm{z}$ for NDGA and $227.2-143.2 \mathrm{~m} / \mathrm{z}$ for resveratrol (internal standard). The column was Hypersil C8 
(4.6x100 mm, $5 \mu \mathrm{m}$ particle size) with mobile phase consisting of $40 \%$ acetonitrile containing $0.05 \%$ of acetic acid. The precision, defined by the coefficient of variation (CV), [(standard deviation (SD)/mean) x 100] of quality control (QC) samples at 3 different drug concentrations $(80.0,15.0$ and $3.00 \mathrm{ng} / \mathrm{ml})$, ranged from 5.80 to $10.4 \%$. Accuracy, defined by the relative error (RE) [(mean - nominal concentration)/nominal concentration) $\mathrm{x} 100$ ] of QC samples, ranged from -5.38 to $-1.00 \%$. The standard curve CVs ranged from 1.77 to $9.70 \%$ and REs ranged from -9.38 to $+7.00 \%$. The average extraction recovery at three concentrations $(80.0,15.0$ and $3.00 \mathrm{ng} / \mathrm{ml})$ was $87.9 \%$. NDGA in human plasma at two concentrations (80.0 and $3.00 \mathrm{ng} / \mathrm{ml}$ ) was shown to be stable for at least 3 freeze/thaw cycles when samples were frozen to $-70^{\circ} \mathrm{C}$ and thawed to room temperature, stable for at least $4 \mathrm{~h}$ when kept on ice water, and stable for $>2$ months when stored at $-70^{\circ} \mathrm{C}$.

Statistical considerations. The primary study end-point was the PSA response proportion according to consensus criteria. (21) With a 0.05 level of significance for a directional test and power of 0.81 , a sample size of 28 patients was required to detect a $35 \%$ PSA response proportion for subjects treated with NDGA compared with a null hypothesis of $15 \%$. A twostage design for accrual was employed to carry out an interim analysis for lack of efficacy. If at most 1 PSA response was observed among the first 12 patients, then accrual would stop and the trial would close for futility. In addition, if NDGA or its metabolites were not detectable in the serum of any of the first 12 patients during the initial 8-h analysis or the first 28-day analysis, the study would not continue to accrue. The probability of stopping accrual at the first stage of accrual if the null hypothesis was true was 44 and $4 \%$ if the alternative hypothesis was true. If accrual was completed, then the null hypothesis of a $15 \%$ response proportion would be rejected if at least 8 of 28 patients achieved a PSA response. Descriptive statistics (e.g. proportion, median, range) were used to characterize the baseline patient and disease features. Due to the small sample size $(n=12)$ non-parametric methods were used for analysis to evaluate the change in testosterone, dihydrotestosterone and androstenedione over time with therapy without any adjustment for multiple comparisons. The Wilcoxon-matched pairs test was used to compare the baseline measurements with the start of each of the next three cycles of protocol therapy for each of the androgen parameters. $\mathrm{p}<0.05$ was considered statistically significant. PSADT was calculated using the formula natural $\log 2$ divided by the slope of the natural $\log$ of the PSA versus time. Pretreatment PSADT was calculated using a minimum of 3 values and end of study PSADT incorporated all measured PSA values on study starting at C2D1 until the patient was removed from study. The Spearman rank-order correlation coefficients were calculated to summarize the relationships between the change in PSA and PSADT with the change in hormone levels. Analyses were performed using Statistica (v6, StatSoft, Inc.).

\section{Results}

Patient characteristics. Table 1 lists pertinent baseline characteristics of enrolled patients. The median age was 67.5 years (range 55-86). Eight patients $(67 \%)$ had a prior radical prosta-
Table I. Baseline characteristics.

No. of patients

Median age (range) in years

$67.5(55-86)$

Primary therapy n $(\%)$

Prostatectomy

Radiation therapy

Salvage radiation therapy

post-prostatectomy

Median PSA ng/ml (range)

$5.1(1.5-9.1)$

Median months PSADT

$8.2(6.7-15.4)$

Median Gleason score

Median gm/dl hemoglobin

$15.5(12.9-16.5)$

Median U/l alkaline phosphatase

$36.5(33-89)$

Median Karnofsky performance status

$90(80-100)$

Median ng/dl testosterone

$353.0(241-521)$

Median ng/dl dihydrotestosterone

$30.5(11-333.6)$

Median ng/dl androstenedione

$43.0(0.8-81)$

tectomy and 4 patients $(33 \%)$ had primary radiation therapy. All patients had PSA-only recurrence. The median PSA for enrolled patients was $5.1 \mathrm{ng} / \mathrm{ml}$ (range 1.5-9.1), median baseline PSADT was 8.2 months (6.7-15.4), median hemoglobin was $15.5 \mathrm{~g} / \mathrm{dl}$ (12.9-16.5), median alkaline phosphatase was $36.5 \mathrm{U} / 1$ (33-89), median Gleason score was 7 (6-9), and all patients except one had a Karnofsky performance status $>90$. The median baseline testosterone level was $353 \mathrm{ng} / \mathrm{dl}$ (241-521), the median baseline dihydrotestosterone level was $30.5 \mathrm{ng} / \mathrm{dl}$ (11.0-333.6) and the median baseline androstenedione level was $43 \mathrm{ng} / \mathrm{dl}(0.8-81)$. There were no significant correlations between the baseline hormone levels measured, nor between baseline hormone levels and baseline PSA.

Patient disposition and response to therapy. Median time on treatment was 9 cycles (range 2-19) for 11 patients now off study and one patient currently remains on study therapy having received 29 cycles of treatment. All patients except one maintained a $>250 \mathrm{ng} / \mathrm{dl}$ level of testosterone throughout the study. Five patients $(42 \%)$ discontinued therapy due to PSA progression. Four patients (33\%) withdrew consent in response to a rising PSA not meeting pre-defined criteria for progression, 1 patient $(8 \%)$ was taken off study for non-compliance with study therapy, and $1(8 \%)$ patient discontinued therapy due to toxicity (recurrent grade 2 weakness) at the end of cycle eleven. No objective disease progression was found on end of study imaging among any of the 11 patients who came off study, however one patient was found to have a biopsy-proven nonsmall cell lung cancer.

PSA responses. Seven patients in total experienced a decline in PSA during the first 3 cycles, ranging from 1.9 to $15.8 \%$ of baseline (Fig. 1); there were no late PSA responses observed. Despite this, no declines in PSA meeting consensus criteria were observed in the first 12 patients and accrual was stopped due to pre-specified stopping criteria for futility. At the time of evaluation of response at the end of cycle 3 the median PSA 


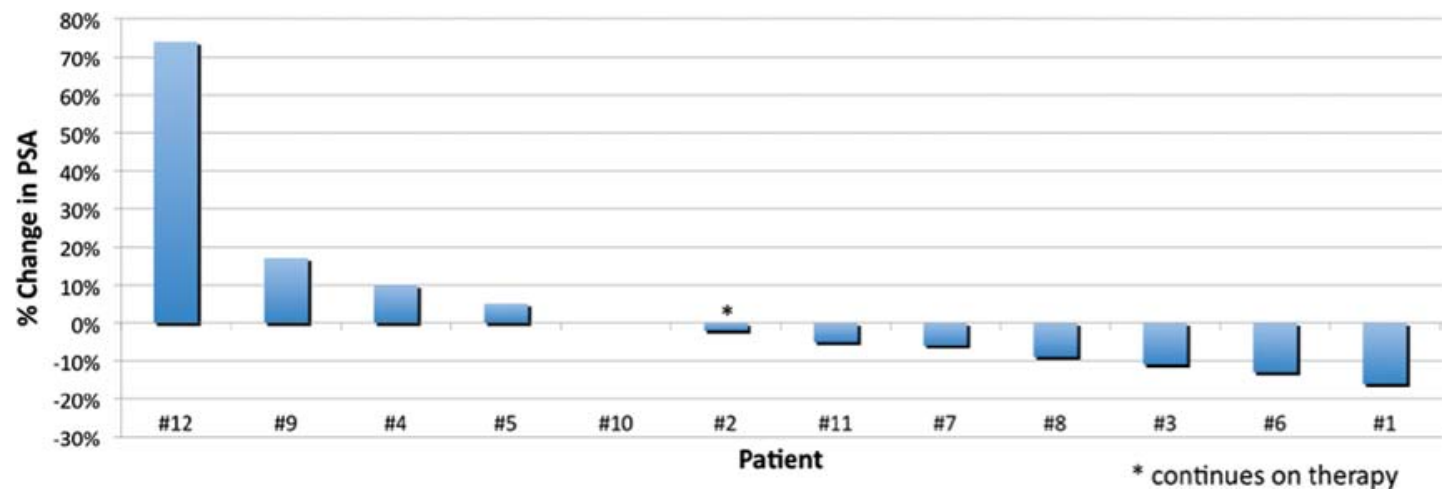

Figure 1. PSA responses.

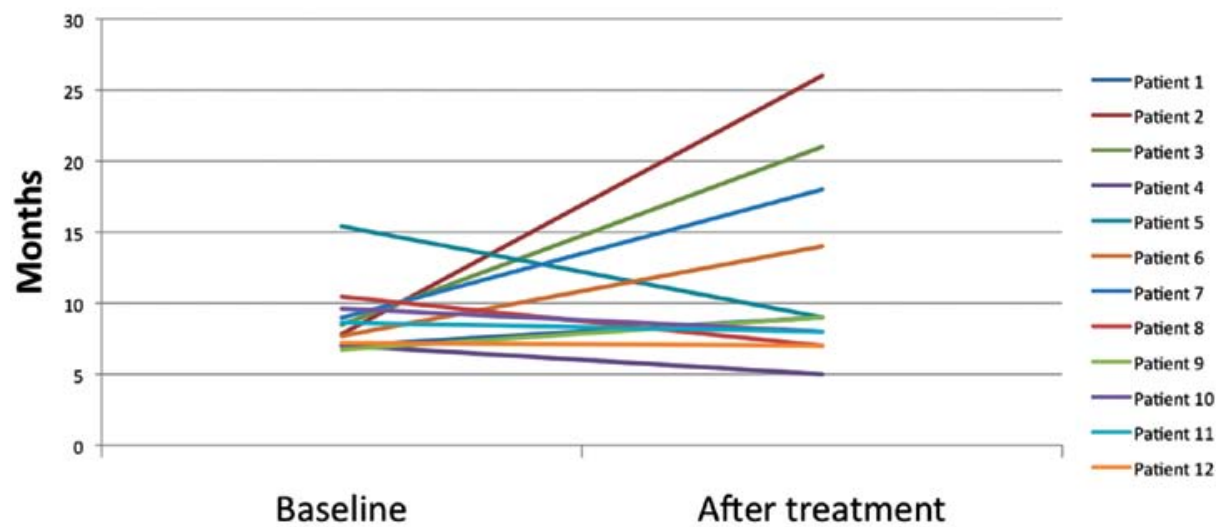

Figure 2. Change in PSA doubling time.

for all patients increased by $34 \%$ (range -12.8 to +49.0 ) over baseline.

Despite the lack of significant PSA responses, the median change in PSADT between the end of study and pretreatment increased by 1.4 months $(-6.1$ to +19.8$)$ for all evaluable patients including the one patient still on study, corresponding to a median $17 \%$ (-66 to $+72 \%$ ) increase in PSADT (Fig. 2). Specifically, the PSADT lengthened in 6 patients when compared to pretreatment PSADT (50\%; 95\% confidence interval 21-79\%); in this subgroup where some improvement was observed, the median increase was 6.9 months (range: 1.4-19.8 months), representing a median $45.3 \%$ increase in the PSADT over baseline. Five of these 6 subjects also had PSA declines at some point on study. In four of these subjects the PSADT increased from $<12$ months before study therapy to $>12$ months at the time of study discontinuation.

Pharmacokinetic evaluation. Pharmacokinetics were evaluated for all 12 patients. After the first 750-mg dose on study the parent drug plasma levels of NDGA were detectable at all time points over $8 \mathrm{~h}$ in the serum of only one patient $(8 \%)$, with $\mathrm{C}_{\max }=8.7 \mathrm{ng} / \mathrm{ml}$. Seven other patients $(58 \%)$ had at least one quantifiable level above the $1 \mathrm{ng} / \mathrm{ml}$ limit of quantitation (LOQ) after the first dose; the median quantifiable $\mathrm{C}_{\max }$ in these patients was $1.6 \mathrm{ng} / \mathrm{ml}(1.1-2.2)$. The remaining 4 patients (33\%) had levels below the LOQ; the median estimated $\mathrm{C}_{\max }$ in this group was $0.49 \mathrm{ng} / \mathrm{ml}(0.42-0.8)$. Because of this neither the maximum drug concentrations $\left(\mathrm{C}_{\max }\right)$, area under the curve, interpatient variability, apparent volume of distribution, nor terminal half-life were able to be determined. GlucoronidatedNDGA metabolites were detected, however a detailed analysis of the metabolite was not carried out due to the absence of a reference standard.

Because of the very low observed levels, the question was explored of whether degradation of NDGA occurred during storage. When NDGA was spiked to blank plasma treated with ascorbic acid no significant degradation was found during sample storage and processing. As all clinical samples were treated with ascorbic acid, degradation of NDGA during sample processing and storage was therefore considered unlikely. Similarly, analysis of samples within days of receipt failed to detect NDGA better than analysis of samples stored for longer periods of time, again suggesting that degradation of NDGA during storage did not occur.

Hormone levels. Eleven of 12 patients had at least 1 followup assessment for testosterone and 10 of the 12 had at least 1 follow-up assessment for DHT and androstenedione. Overall seven patients each experienced declines in testosterone, DHT and androstenedione while on study; four patients experienced declines in all 3 hormone levels. The median maximum percent change in hormone levels for the entire study cohort was $-18 \%$ 


\section{DHT Response vs PSADT Response}

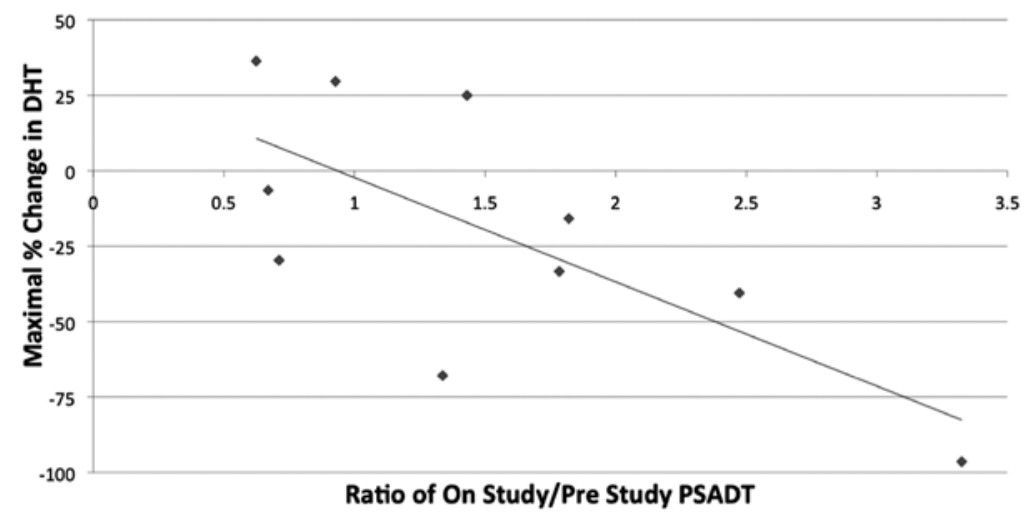

Figure 3. Lower DHT values were observed in patients who experienced longer PSA doubling times.

(range -39.8 to 0 ) for testosterone, $-15.8 \%$ ( -96.4 to 36.4 ) for DHT, and $-20.7 \%$ (-48.6 to 0 ) for androstenedione.

All patients who experienced lengthening in the PSADT had a decrease at some point in at least one of the hormone levels measured. The correlation between decline in hormone levels and PSADT response was not uniform, however; of the 4 patients who experienced declines in all 3 hormone levels measured, only 3 had lengthening in PSADT. Patients achieving a decline in DHT on study were more likely to have a lengthening in PSADT (percent change: DHT versus PSADT: Spearman correlation $-0.64, p=0.05)$, however no other correlations between change in hormone levels and change in PSADT were observed (Fig. 3).

Toxicity. Among 12 patients a total of 66 adverse events occurred (Table II). Two patients experienced CTC-defined grade 3 events (nausea, syncope and elevated AST in one patient and cognitive disturbance in another patient) that were classified as possibly related to NDGA. These grade 3 toxicities all resolved to grade 1 or less with discontinuation of NDGA. The most common events included grade 1 or 2 diarrhea responsive to treatment with anti-motility agents in all patients (100\%), elevated aspartate or alanine transaminase (AST or ALT) in 8 patients $(67 \%)$, and grade 1 fatigue in 5 cases $(42 \%)$ that were all felt to be likely related to therapy. Overall the therapy was well tolerated with no grade 4 or higher toxicities reported.

New elevations in hepatic transaminases were observed at the outset of the study including one grade 3 elevation in AST. Some subjects (nos. 1,2,10) experienced multiple recurrences of grade 1 or 2 elevations in AST or ALT which resolved without discontinuation of study therapy.

\section{Discussion}

This study evaluated whether NDGA could lower PSA in men with biochemically relapsed hormone-sensitive prostate cancer and normal testosterone levels, and it was hypothesized that impairment of intratumoral IGF signaling would lead to improvement in markers of clinical response. Consistent with both preclinical data and previous clinical results, half of the patients receiving NDGA experienced a prolongation in their
PSADT $(15,17)$. Despite this lengthening, however, there were no PSA responses meeting predefined consensus criteria observed in this study.

There are a number of potential reasons explaining the lack of significant PSA declines in this group of patients. First, the pharmacokinetic analysis in this study failed to consistently detect NDGA above the LOQ in the majority of patients. The fact that degradation of NDGA during sample processing and storage was excluded, that NDGA glucoronide metabolites were detected, and that liver function test (LFT) abnormalities were observed in multiple patients (indicating delivery of drug to hepatocytes), suggest that first-pass hepatic metabolism, as opposed to low absorption, resulted in the low observed bioavailibility of NDGA. This finding of efficient first-pass metabolism has been observed with other polyphenols as well $(24,25)$. While a $2500-\mathrm{mg} /$ day dose of NDGA has previously been shown to be safe and well tolerated (17), it is not known whether this dose would in fact have better bioavailability. Additionally as NDGA levels were consistently detectable in only 1 patient (13\%), it is possible that there exist germline variations in drug metabolism which contribute to the difficulty in detecting drug levels. Whether it is possible to select patients who metabolize the parent drug more slowly is unknown.

Second, because patients had no measurable disease at the start of the study, it is not known to what degree the IGFR1 was expressed on cancer cells in this unselected patient population. Similarly, in men who had detectable NDGA levels, it is unknown to what degree the IGFR1 was inhibited. Inhibitors of IGF signaling have been associated with hyperglycemia (26), however hyperglycemia (grade 1) was observed in only 1 patient, suggesting that NDGA may not have reached the IGFR1 target. The fact that men in this study had no measurable disease precluded on-study biopsy to look for other downstream markers of IGFR1 activation; similarly, except for serum glucose levels, there are no established pharmacodynamic markers to evaluate to what degree IGFR1 signaling is inhibited in vivo. While a compensatory rise in circulating IGF levels or circulating growth hormone levels could be postulated to provide indirect evidence of receptor target inhibition, these were not assayed in this study. 
Table II. Toxicities by CTC version 3.0.

\begin{tabular}{|c|c|c|c|}
\hline Event & $\begin{array}{l}\text { CTC grade } 1 \\
\text { No. pts. }(\%)\end{array}$ & $\begin{array}{l}\text { CTC grade } 2 \\
\text { No. pts. }(\%)\end{array}$ & $\begin{array}{l}\text { CTC grade } 3 \\
\text { No. pts. }(\%)\end{array}$ \\
\hline Abdominal distension & $2(16)$ & $1(8)$ & 0 \\
\hline Cognitive disturbance & 0 & 0 & $1(8)$ \\
\hline Dehydration & 0 & $1(8)^{\mathrm{a}}$ & 0 \\
\hline Diarrhea & $11(92)^{\mathrm{a}}$ & $1(8)^{\mathrm{a}}$ & 0 \\
\hline Dry mouth & $1(8)$ & 0 & 0 \\
\hline Dyspnea & $1(8)$ & 0 & 0 \\
\hline Elevated alkaline phosphate & 0 & $2(16)$ & 0 \\
\hline Elevated ALT & $6(50)^{\mathrm{a}}$ & $2(16)^{\mathrm{a}}$ & 0 \\
\hline Elevated AST & $5(42)^{\mathrm{a}}$ & 0 & $1(8)^{\mathrm{a}}$ \\
\hline Elevated bilirubin & $2(16)^{\mathrm{a}}$ & 0 & $1(8)^{\mathrm{a}}$ \\
\hline Elevated CPK & $1(8)$ & 0 & 0 \\
\hline Elevated GGT & 0 & 0 & $1(8)$ \\
\hline Fatigue & $5(42)^{\mathrm{a}}$ & 0 & 0 \\
\hline Fever & 1 (8) & 0 & 0 \\
\hline Gastritis & $1(8)$ & 0 & 0 \\
\hline Headache & $4(33)^{a}$ & 0 & 0 \\
\hline Hyperglycemia & $1(8)$ & 0 & 0 \\
\hline Hypoalbuminemia & 0 & $1(8)^{\mathrm{a}}$ & 0 \\
\hline Hypocalcemia & 0 & $1(8)$ & 0 \\
\hline Insomnia & $1(8)$ & 0 & 0 \\
\hline Leukopenia & 0 & $1(8)$ & 0 \\
\hline Muscle weakness & 0 & $1(8)$ & 0 \\
\hline Myalgia & $1(8)$ & 0 & 0 \\
\hline Nausea & $2(16)^{a}$ & 0 & $1(8)^{\mathrm{a}}$ \\
\hline Rash & $1(8)$ & 0 & 0 \\
\hline Syncope & 0 & 0 & $1(8)$ \\
\hline Visual disturbance & $1(8)$ & 0 & 0 \\
\hline Vomiting & $1(8)$ & 0 & 0 \\
\hline Weight loss & 0 & $1(8)$ & 0 \\
\hline
\end{tabular}

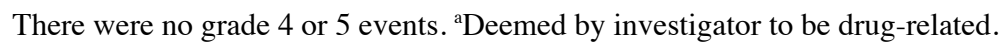

A third explanation for the lack of efficacy is that the possibility that any growth inhibitory effect of NDGA was overcome by intact androgen signaling through the AR. Supporting this notion is the fact that PSADT lengthening was correlated with decline in DHT, although the precise cause of variations in DHT levels over time is unknown. It is possible that IGF signaling is more important in more advanced disease; a study evaluating the effect of a biologically available dose of NDGA used in conjunction with androgen deprivation therapy or given after the development of castration resistance could better address this question.

Despite the fact that no PSA declines meeting predefined criteria were observed, the majority of subjects had a decline in PSA at some point on the study and half of subjects experienced a clinically relevant prolongation in their PSADT with a median change of 6.9 months compared to PSADT at study entry (Figs. 1 and 2). This finding was also observed in earlier studies (17). Thus, despite the observed correlation with DHT declines, it is still possible that that the low biologically available doses of NDGA were effective at slowing growth, or that the metabolites of NDGA have some modest biologic efficacy. In order to further study this effect a parenteral formulation could be tested, which would potentially avoid the problem of first-pass hepatic metabolism and could lead to more consistently detectable levels of the parent drug. Alternatively, a higher dose of oral NDGA could be given. Lastly, receptor inhibition with NDGA could be combined with therapies directed at reducing circulating IGF levels, such as octreotide acetate, in order to better disrupt the IGF signaling pathway.

In this study NDGA was well tolerated. Diarrhea and elevation in hepatic transaminases were the most common side effects, however they were mild (CTC grade 2 or less) in the majority of patients. Diarrhea in all cases was amenable to supportive care and in all cases but one LFT abnormalities resolved spontaneously without study drug discontinuation. As patients in this study received therapy for a median of 36 weeks, the long- 
term effects of this therapy remain unknown. Similarly, as IGF signaling is thought to be a key mediator of cell growth and survival, it is not known if long-term suppression of IGF signaling with NDGA would place men with prostate cancer at increased risk of developing problems with bone or cardiac health.

In summary, this study was conducted to evaluate whether IGFR1 inhibition with NDGA could slow the growth of biochemically relapsed hormone-sensitive prostate cancer, allowing men to avoid many of the deleterious side-effects of androgen deprivation therapy. Although treatment with oral NDGA is associated with prolongations in the PSADT in some men, it does not result in PSA responses and cannot be recommended as monotherapy in this population. Because unanticipated fluctuations in DHT levels may have contributed to this lengthening, a dedicated placebo-controlled study with a larger sample size and a biologically detectable dose, using PSADT as a primary endpoint, would help to clearly define the effect of NDGA in this population. Selecting subjects based on tumor IGFR1 expression could help enrich the study with patients more likely to respond. Monitoring of biomarkers of IGF1R inhibition, such as glucose, circulating IGF, or circulating IGF binding protein levels, could also help better define the NDGA's in vivo effect on the IGF1R, and potentially could help better identify the patient subset(s) most likely to respond. Consideration could also be given to adding a biologically detectable dose to androgen deprivation therapy for men with more advanced disease.

\section{Acknowledgements}

This study was supported by NIH K23 grant CA115775 (C.J.R.) and the John Kerner fund. The authors would like to gratefully acknowledge the contributions of Dr Ira Goldfine and Dr John Kerner.

\section{References}

1. Fottner C, Hoeflich A, Wolf E and Weber MM: Role of the insulin-like growth factor system in adrenocortical growth control and carcinogenesis. Horm Metab Res 36: 397-405, 2004.

2. Moschos SJ and Mantzoros CS: The role of the IGF system in cancer: from basic to clinical studies and clinical applications. Oncology 63: 317-332, 2002.

3. Sell C, Baserga R and Rubin R: Insulin-like growth factor I (IGF-I) and the IGF-I receptor prevent etoposide-induced apoptosis. Cancer Res 55: 303-306, 1995.

4. Wu Y, Tewari M, Cui S and Rubin R: Activation of the insulin-like growth factor-I receptor inhibits tumor necrosis factor-induced cell death. J Cell Physiol 168: 499-509, 1996.

5. LeRoith D, Werner H, Beitner-Johnson D and Roberts CT Jr: Molecular and cellular aspects of the insulin-like growth factor I receptor. Endocr Rev 16: 143-163, 1995.

6. Riedemann J and Macaulay VM: IGF1R signalling and its inhibition. Endocr Relat Cancer 13 (Suppl 1): S33-S43, 2006.

7. Kaplan PJ, Mohan S, Cohen P, Foster BA and Greenberg NM: The insulin-like growth factor axis and prostate cancer: lessons from the transgenic adenocarcinoma of mouse prostate (TRAMP) model. Cancer Res 59: 2203-2209, 1999.
8. Wolk A, Mantzoros CS, Andersson SO, et al: Insulin-like growth factor 1 and prostate cancer risk: a population-based, casecontrol study. J Natl Cancer Inst 90: 911-915, 1998.

9. Culig Z, Hobisch A, Cronauer MV, et al: Androgen receptor activation in prostatic tumor cell lines by insulin-like growth factor-I, keratinocyte growth factor, and epidermal growth factor. Cancer Res 54: 5474-5478, 1994.

10. Burfeind P, Chernicky CL, Rininsland F and Ilan J: Antisense RNA to the type I insulin-like growth factor receptor suppresses tumor growth and prevents invasion by rat prostate cancer cells in vivo. Proc Natl Acad Sci USA 93: 7263-7268, 1996.

11. Cardillo MR, Monti S, Di Silverio F, Gentile V, Sciarra F and Toscano V: Insulin-like growth factor (IGF)-I, IGF-II and IGF type I receptor (IGFR-I) expression in prostatic cancer. Anticancer Res 23: 3825-3835, 2003.

12. Arteaga S, Andrade-Cetto A and Cardenas R: Larrea tridentata (Creosote bush), an abundant plant of Mexican and US-American deserts and its metabolite nordihydroguaiaretic acid. J Ethnopharmacol 98: 231-239, 2005.

13. Zavodovskaya M, Campbell MJ, Maddux BA, et al: Nordihydroguaiaretic acid(NDGA), an inhibitor of the HER2 and IGF-1 receptor tyrosine kinases, blocks the growth of HER2-overexpressing human breast cancer cells. J Cell Biochem 103: 624-635, 2008.

14. Youngren JF, Gable K, Penaranda C, et al: Nordihydroguaiaretic acid (NDGA) inhibits the IGF-1 and c-erbB2/HER $2 /$ neu receptors and suppresses growth in breast cancer cells. Breast Cancer Res Treat 94: 37-46, 2005.

15. Ryan CJ, Zavodovskaya M, Youngren JF, et al: Inhibitory effects of nordihydroguaiaretic acid (NDGA) on the IGF-1 receptor and androgen dependent growth of LAPC-4 prostate cancer cells. Prostate 68: 1232-1240, 2008.

16. Huang JK, Chen WC, Huang CJ, et al: Nordihydroguaiaretic acid-induced $\mathrm{Ca} 2+$ handling and cytotoxicity in human prostate cancer cells. Life Sci 75: 2341-2351, 2004.

17. Ryan CJ, Harzstark AH, Rosenberg J, et al: A pilot doseescalation study of the effects of nordihydroguareacetic acid on hormone and prostate specific antigen levels in patients with relapsed prostate cancer. BJU Int 101: 436-439, 2008.

18. Keating NL, O'Malley AJ, Freedland SJ and Smith MR: Diabetes and cardiovascular disease during androgen deprivation therapy: observational study of veterans with prostate cancer. J Natl Cancer Inst 102: 39-46, 2010.

19. Lattouf JB, Fadlallah H and Saad F: Androgen deprivation and bone. Curr Osteoporos Rep 9: 20-24, 2011.

20. Roach M 3rd, Hanks G, Thames H Jr, et al: Defining biochemical failure following radiotherapy with or without hormonal therapy in men with clinically localized prostate cancer: recommendations of the RTOG-ASTRO Phoenix Consensus Conference. Int J Radiat Oncol Biol Phys 65: 965-974, 2006.

21. Bubley GJ, Carducci M, Dahut W, et al: Eligibility and response guidelines for phase II clinical trials in androgen-independent prostate cancer: recommendations from the Prostate-Specific Antigen Working Group. J Clin Oncol 17: 3461-3467, 1999.

22. Trotti A, Colevas AD, Setser A, et al: CTCAE v3.0: development of a comprehensive grading system for the adverse effects of cancer treatment. Semin Radiat Oncol 13: 176-181, 2003.

23. United States Department of Health and Human Services, Food and Drug Administration (FDA), Center for Drug Evaluation and Research (CDER): Guidelines for Industry: Food-Effect Bioavailability and Fed Bioequivalence Studies. http://www.fda. gov/cder/guidance/index.htm.

24. Manach C, Scalbert A, Morand C, Remesy C and Jimenez L: Polyphenols: food sources and bioavailability. Am J Clin Nutr 79: 727-747, 2004

25. Walle T, Hsieh F, DeLegge MH, Oatis JE Jr and Walle UK: High absorption but very low bioavailability of oral resveratrol in humans. Drug Metab Dispos 32: 1377-1382, 2004.

26. Crouthamel MC, Kahana JA, Korenchuk S, et al: Mechanism and management of AKT inhibitor-induced hyperglycemia. Clin Cancer Res 15: 217-225, 2009. 